\title{
DEFECTS OF NON-STOICHIOMETRY AND DYNAMIC STABILITY OF SnTe CRYSTAL LATTICE
}

\author{
E.I. Rogacheva, N.A. Sinelnik, O.N. Nashchekina \\ Polytechnical Institute, 21, Frunze St., Kharkov 310002, Ukraine \\ V.P. Popov and T.A. LoBkovskaya \\ Kharkov State University, 4, Svoboda Sq., Kharkov 310077, Ukraine
}

\begin{abstract}
The temperature dependencies of coefficient of linear expansion $\alpha$ in the range of 4.2-300 K were obtained for SnTe samples with various degrees of deviation from stoichiometry. For the sample of stoichiometric composition in the $\alpha$ vs. temperature curves the anomalies connected with ferroelectric phase transition were detected. It was found that within cubic phase existence region $\alpha$ isotherms have a minimum at 50.4 at.\% Te. Maximum dynamic stability at indicated composition is believed to be associated with the formation of vacancy superstructure.
\end{abstract}

PACS numbers: $61.70 . \mathrm{Bv}, 65.70 .+\mathrm{y}$

As is generally known, all the semiconducting compounds are characterized by one or another degree of deviation from stoichiometry. Therefore, studying the effect of non-stoichiometric defects on physical properties, crystal and energy band structure of compounds and alloys is related to the most important problems of semiconductor material science.

The goal of the present investigation was to study the influence of non-stoichiometric vacancies on dynamic properties of crystal, in particular on coefficient of linear expansion $\alpha$ being the characteristic of atom oscillation anharmonicity degree. The object of study was semiconducting compound SnTe which crystallizes in the $\mathrm{NaCl}$-type lattice and has a wide $(\approx 1$ at.\%) single-sided homogeneity region located on the side of the Te excess (the peak in melting curves in $\mathrm{Sn}-\mathrm{Te}$ system corresponds to a composition 50.4 at.\% Te) [1]. This fact determines a great amount of intrinsic defects, mainly cation vacancies in $\mathrm{SnTe}$ and, consequently, high concentration of $p$-type charge carriers $\left(\approx 10^{20}-10^{21} \mathrm{~cm}^{-3}\right)$ [2]. It has been reported $[3,4]$ about ferroelectric phase transition in SnTe accompanied by rhombohedral distortion of crystal lattice.

In this work we investigated tin telluride samples with Te contents of 50.0-51.0 at.\% (the boundaries of homogeneity region of SnTe were $50.1-50.8$ at.\% $\mathrm{Te}$ ) which correspond to different vacancy concentrations in cation 
sublattice (0.4-3.2 at.\%). The temperature dependencies of $\alpha$ in the temperature range of 5-300 K were obtained using differential dilatometer with optical-mechanical registration of thermal elongation on the samples prepared by hot pressing from polycrystalline bulks.

For the samples with Te content of 50.0 and 50.1 at.\% in $\alpha$ vs. temperature curves in the vicinity of $90 \mathrm{~K}$ there was noticed a pronounced anomaly (Fig. 1), associated with the phase transition. For the remaining samples the anomaly was

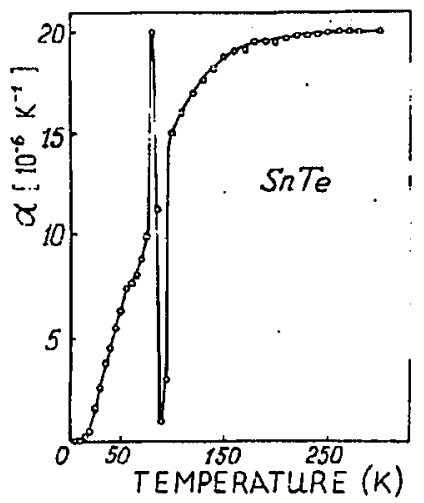

Fig. 1. Temperature dependence of coefficient of linear expansion in SnTe.

not observed which agreed with dependence of ferroelectric transition temperature on Te content in the homogeneity region of SnTe [5]. In the range of 5-100 K the lowest $\alpha$ value belonged to the samples with 50.0 and 50.1 at.\% $\mathrm{Te}$, which was probably associated with their crystallization in rhombohedral structure. Let us note that similar picture has been observed in GeTe [1]: for $\alpha$-GeTe with rhombohedral structure $\alpha$ is much lower than for $\beta$-GeTe with cubic lattice. After reaching the Debye temperature $\left(\Theta_{\mathrm{D}} \approx 130 \mathrm{~K}[1]\right)$ linear expansion coefficient $\alpha$ of studied samples changes insignificantly. The coefficient $\alpha$ vs. temperature curves of different samples crystallizing in the same $\mathrm{NaCl}$ structure are virtually parallel. The lowest value of $\alpha$ belongs to the sample with 50.4 at.\% Te. As a result, the isotherms of $\alpha$ represent curves with minimum at 50.4 at.\% Te (Fig. 2).

It has been reported by other authors previously [6] that introduction of impurity atoms or other defects leads to the increase in $\alpha$ of metals and semiconductors. This is in good agreement with theoretical calculation results which attribute changing of $\alpha$ both to changing configurational potential energy of ideal crystal at introduction of impurity atoms and other defects and to influence of free charge carriers on heat capacity and Gruneisen parameter [7]. However, such a character of the dependence is apparently peculiar to diluted solid solutions. The interactions between the defects can qualitatively change the character of the dependence. Taking into consideration the high concentration of cation vacancies, one can suggest that decrease in $\alpha$ with increase in the vacancy concentration is caused by the interaction between vacancies which induces their redistribution, for instance, formation of vacancy superstructure. The fact that non-stoichiometric 


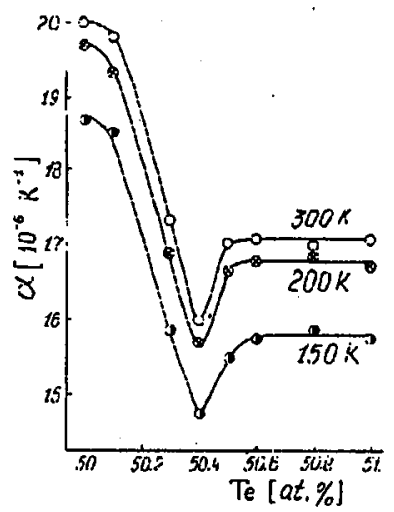

Fig. 2. Coefficient of linear expansion of tin telluride vs. Te content at various temperatures.

vacancies in $\mathrm{SnTe}$ are charged supports the idea of their ordering. According to theoretical investigations [11] the Coulomb interaction of impurity centers results in forming of superstructure of ionized defects. The latter supposition is confirmed by presence of extremum on the concentration dependencies of the Mössbauer parameter, diffraction peak width and other properties at 50.4 at.\% Te we have observed earlier [8-10].

Let us denote the average distance between vacancies as $d$. Then the formation of vacancy fcc lattice with unit-cell parameter $a=2 n a_{0}$, where $a_{0}$ is $\mathrm{SnTe}$ unit-cell parameter and $n$ is integer, corresponds to the condition $d=2 n a_{0} / \sqrt[3]{4}$. Simple calculation shows that it is at 50.4 at.\% Te that formation of vacancy fcc lattice with $a=4 a_{0}$ is possible.

It is known [6] that $\alpha$ can be expressed as $\alpha=\beta k / \varepsilon^{2} \tau_{0}$, where $\varepsilon$ is quasielastic bond coefficient; $\beta$ is coefficient of anharmonicity; $\tau_{0}$ denotes the distance between atoms; $k$ is the Boltzmann constant. Although the formula is only approximate and correct for two-atom model in case that crystal structure remains unchanged one can perform comparative estimations of crystal anharmonicity degree which is characterized by magnitude of $\beta / \varepsilon^{2}$.

From $\alpha$ value one can estimate as well the average displacement of atoms from equilibrium positions $\bar{x}$ proportional to temperature: $\bar{x}=\alpha \tau_{0} T$. The calculation shows that it is approximately equal to $0.006 \tau_{0}$ for $\mathrm{SnTe}$ at $300 \mathrm{~K}$. Table lists the values of $\alpha, \beta / \varepsilon^{2}, \bar{x}$ for Sn-Te alloys with the different content of non-stoichiometric vacancies at $300 \mathrm{~K}$. As it is seen from Table the increase of vacancy concentration in cation sublattice from 0.4 up to $1.6 \%$ results in decreasing $\alpha, \beta / \varepsilon^{2}, \bar{x}$ by $\approx 20-25 \%$.

There is a simple relation between the coefficient of linear expansion and the Debye temperature expressed by formula [6]:

$$
\Theta_{\mathrm{D}}=\sqrt{A / \alpha V^{2 / 3} M}
$$


TABLE

Dynamic parameters of $\mathrm{Sn}-\mathrm{Te}$ alloys.

\begin{tabular}{c|c|c|c|c}
\hline \hline Te [at. \%] & $\tau_{0} \cdot 10^{10}[\mathrm{~m}]$ & $\alpha \cdot 10^{6}\left[\mathrm{~K}^{-1}\right]$ & $\beta / \varepsilon^{2} \cdot 10^{8}\left[\mathrm{~N}^{-1}\right]$ & $\bar{x} \cdot 10^{12}[\mathrm{~m}]$ \\
\hline 50.0 & 6.324 & 20 & 9.16 & 3.79 \\
50.4 & 6.314 & 16 & 7.3 & 3.03 \\
51.0 & 6.300 & 17 & 7.8 & 3.2
\end{tabular}

where $M$ is molecular weight, $A$ is constant depending upon chemical bond type. From $\alpha$ values one can approximately estimate change of $\Theta_{D}$ on shifting from stoichiometric composition to 50.4 at.\% $\mathrm{Te}(\approx 10 \%)$. The evaluation of $\Theta_{\mathrm{D}}$ change within SnTe homogeneity region performed on the basis of the Mössbauer data reported in [9] gives $\approx 8 \%$ and this result is consistent with our estimation.

From the data obtained it follows that the alloys with Te content corresponding to maximum fusion temperature and, consequently, the maximum thermal stability within $\mathrm{SnTe}$ homogeneity region have the maximum dynamical stability.

\section{References}

[1] N.Kh. Abrikosov, L.Ye. Shelimova, Semiconducting Materials Based on $A^{I V} B^{V I}$ Compounds, Nauka, Moscow 1975.

[2] R.F. Brebrick, J. Phys. Chem. Solids 24, 27 (1963).

[3] H. Kawamura, Comments Solid State Phys. 9, 55 (1979).

[4] P.B. Littlewood, Lect. Notes Phys. 152, 238 (1982).

[5] K.L.J. Kobayashi, Y. Kato, Y. Katayama, K.F. Komatsubara, Phys. Rev. Lett. 37, 772 (1976).

[6] S.I. Novikova, Thermal Expansion of Solids, Nauka, Moscow 1974.

[7] A.Z. Varisov, A.I. Rezanov, R.M. Sabitov, Izv. Vyssh. Uchebn. Zaved. Fiz. (USSR) 5, 147 (1966).

[8] D. Baltrunas, S. Motiejunas, E.I. Rogacheva, Phys. Status Solidi A 97, K131 (1986).

[9] E.I. Rogacheva, G.V. Gorne, S.A. Laptev et al., Izv. Akad. Nauk SSSR Neorg. Mater. 22, 41 (1986).

[10] E.I. Rogacheva, G.V. Gorne, N.K. Zhigareva, A.B. Ivanova, Izv. Akad. Nauk SSSR, Neorg. Mater. 27, 267 (1991).

[11] J. Mycielski, Solid State Commun. 60, 165 (1986). 